\title{
Aplicación de la metodología de huella ecológica como indicador de sostenibilidad en el uso de ensilaje de pescado en dietas para alimentación de aves
}

\author{
Yhoan S. Gaviria ${ }^{1}$, Omar A. Figueroa ${ }^{2}$ y José E. Zapata ${ }^{1 *}$ \\ (1) Grupo de investigación en nutrición y tecnología de alimentos, NUTEC, Universidad de Antioquia, Medellín, Colombia \\ (Correo-e: yhoan.gaviria@udea.edu.co; edgar.zapata@udea.edu.co) \\ (2) Facultad de Ingeniería, Universidad de la guajira, Riohacha, Colombia (Correo-e: omfimo22@gmail.com)
}

* Autor a quien debe ser dirigida la correspondencia.

Recibido May. 15, 2021; Aceptado Jul. 19, 2021; Versión final Jul. 30, 2021, Publicado Oct. 2021

\begin{abstract}
Resumen
Los principales objetivos del presente estudio son determinar el impacto ambiental del aprovechamiento de vísceras de tilapia roja (Oreochromis spp.) para la obtención de ensilado químico y evaluar su implementación en dietas para especies avícolas mediante la metodología de huella ecológica como indicador de sostenibilidad. La producción piscícola en el mundo ha aumentado con el pasar de los años debido al aumento de la población y al interés de consumidores por este tipo de alimentos. Los resultados muestran que el aprovechamiento de las vísceras reduce el efecto adverso de su vertimiento al ambiente en $16 \%$. Por otra parte, el uso de recursos naturales para el cultivo de materias primas para la elaboración de alimento y la disposición de aguas residuales son las principales fuentes de emisiones ambientales con $39 \%$ y $60 \%$, respectivamente. Se concluye que aprovechar los subproductos de ambas industrias e implementar energías alternativas renovables y eficientes, reducen significativamente la carga ambiental del proceso.
\end{abstract}

Palabras clave: huella ecológica; aves de corral; residuos orgánicos; energía renovable

\section{Application of the ecological footprint methodology as an indicator of sustainability in the use of fish silage in diets for poultry feeding}

\begin{abstract}
The main objectives of the present study are to determine the environmental impact of using red tilapia (Oreochromis spp.) viscera to produce chemical silage and to assess its implementation in poultry diets by using the ecological footprint methodology as a sustainability indicator. Global fish production has increased over the years due to population growth and consumer interest in this type of food. The results show that using viscera to produce chemical silage reduces the adverse effect of its discharge to the environment by $16 \%$. In contrast, the use of natural resources for producing food processing raw materials and wastewater disposal are the main sources of environmental emissions by $39 \%$ and $60 \%$, respectively. It is concluded that taking advantage of viscera by-products and implementing renewable and efficient energy sources in both industries significantly reduces environmental burden.
\end{abstract}

Keywords: ecological footprint; poultry; organic waste; renewable energy 


\section{INTRODUCCIÓN}

La demanda actual de agua, alimentos, infraestructura y energía, que se tiene a nivel global, ejerce presión ecológica sobre los recursos naturales del planeta y conduce en muchos casos a la generación de residuos y la emisión de gases de efecto invernadero (Ahmed y Wang, 2019). Dicha demanda tiene tendencia creciente, con lo que la capacidad para contrarrestar los impactos provocados por la generación de residuos pierde cada vez más terreno y se ve limitada la biocapacidad del planeta (Sharif et al., 2020). Esto ha hecho necesario la implementación cada vez más, de políticas energéticas y ambientales orientadas a la preservación de los recursos naturales (Sharif et al., 2020; Ibidhi et al., 2017), lo que ha llevado al surgimiento de metodologías para evaluar el impacto sobre el ambiente que pueda generar un proceso dado (Syrovátka, 2020).

La metodología de huella ecológica es uno de los indicadores de sostenibilidad más utilizados para la evaluación del impacto ambiental en procesos de optimización, permitiendo analizar cada etapa (Gwehenberger y Narodoslawsky, 2007). En ella se cuantifica el efecto de las actividades antropogénicas sobre el medio ambiente en función de tierra, agua, productos forestales, infraestructura y huella de carbón (Ahmed y Wang, 2019). Establece la cantidad de hectáreas globales de tierra y agua necesarias para mitigar el efecto de los residuos generados o el consumo de los recursos naturales, en un proceso dado (Ahmed y Wang, 2019). Esta metodología genera respuestas comparables, confiables e integras (Ahmed y Wang, 2019; Jóhannesson et al., 2018).

Una de las actividades productivas que han presentado crecimiento sostenido durante las últimas décadas, lo representa la piscicultura, la cual llegó en 2016 a 171 millones ton en el mundo, mientras que en Colombia se estima que presentó un crecimiento del $9 \%$ en el 2018, siendo la tilapia roja (Oreochromis $s p$ ) el $62 \%$ de la producción (Fao, 2016), En dicha industria se descarta alrededor del $65 \%$ en forma de residuos, los cuales en la mayoría de los casos son vertidos inadecuadamente, implicando un fuerte deterioro ambiental (MartínezAlvarez et al., 2015). Teniendo en cuenta que muchos de estos residuos son ricos en proteína, como es el caso de las vísceras (Suarez et al., 2018), surge la alternativa de utilizarlos como fuente de proteína para la obtención de insumos orientados a la industria de alimentación animal. En este sentido, el ensilaje de vísceras de pescado se ha implementado con éxito aportándole valor agregado a este residuo, extendiendo el periodo de conservación y mejorando su composición nutricional (Suarez et al., 2018).

Por su parte, la avicultura es reconocida como una cadena eficiente para producir proteína animal de alta calidad y bajo costo. La carne de pollo y el huevo de gallina han alcanzado el estatus de alimentos completos, sanos y accesibles para millones de personas en todo el mundo (Gomez et al., 2014). Sin embargo, el 95\% del costo de la alimentación de pollos y gallinas, es destinado a satisfacer las necesidades de proteína y energía, siendo la harina de pescado y la torta de soya las principales fuentes de proteína animal y vegetal, respectivamente (Fao, 2016). Sin embargo, estas materias primas no siempre están disponibles en las cantidades requeridas en todos los países, como es el caso de Colombia, país que se ve obligado a importar de grandes productores como Estados Unidos y Brasil (Fao, 2016). Por este motivo, es necesario buscar alternativas de alimentación que permitan disminuir costos, para mejorar la eficiencia y sostenibilidad de estos sistemas productivos (Gomez et al., 2014).

Adicionalmente, la Organización de Naciones Unidas (ONU) el año 2015, estipulo los Objetivos de Desarrollo Sostenible (ODS), el estudio se encuentra asociado al cumplimiento de los objetivos 12 "producción y consumo responsable", 13 "Acción por el clima" y 15 "vida de ecosistemas terrestres". Teniendo en cuenta que a la fecha no existen reportes de literatura que evalúen el impacto ambiental de la utilización de ensilado de pescado en la alimentación animal, el objetivo del presente estudio fue determinar del impacto ambiental del aprovechamiento de vísceras de tilapia roja (Oreochromis Spp.) para la obtención de ensilado químico y su implementación en dietas para especies avícolas, mediante la metodología de huella ecológica como indicador de sostenibilidad.

\section{MATERIALES Y MÉTODOS}

La metodología presenta varias subsecciones, inicialmente se describe del área de estudio, seguido por las suposiciones y limitaciones del estudio, por último, se establece el cálculo del impacto ambiental.

\section{Área de estudio}

El sistema productivo consistió en una granja piscícola productora de tilapia roja (Oreochromis Ssp.) ubicada en el municipio de San Jerónimo, Antioquia-Colombia $\left(6^{\circ} 26^{\prime} 30^{\prime \prime} \mathrm{N} 75^{\circ} 43^{\prime} 40^{\prime \prime} \mathrm{O}\right)$, con el fin de plantear estrategias de aprovechamiento de residuos como las vísceras, para la obtención de ensilado químico y su uso en la alimentación de especies avícolas. Las figuras 1 y 2 representan los múltiples procesos llevados a cabo en el sistema productivo y el proceso tradicional respectivamente, indicando las salidas de productos y residuos en cada uno de ellos. 


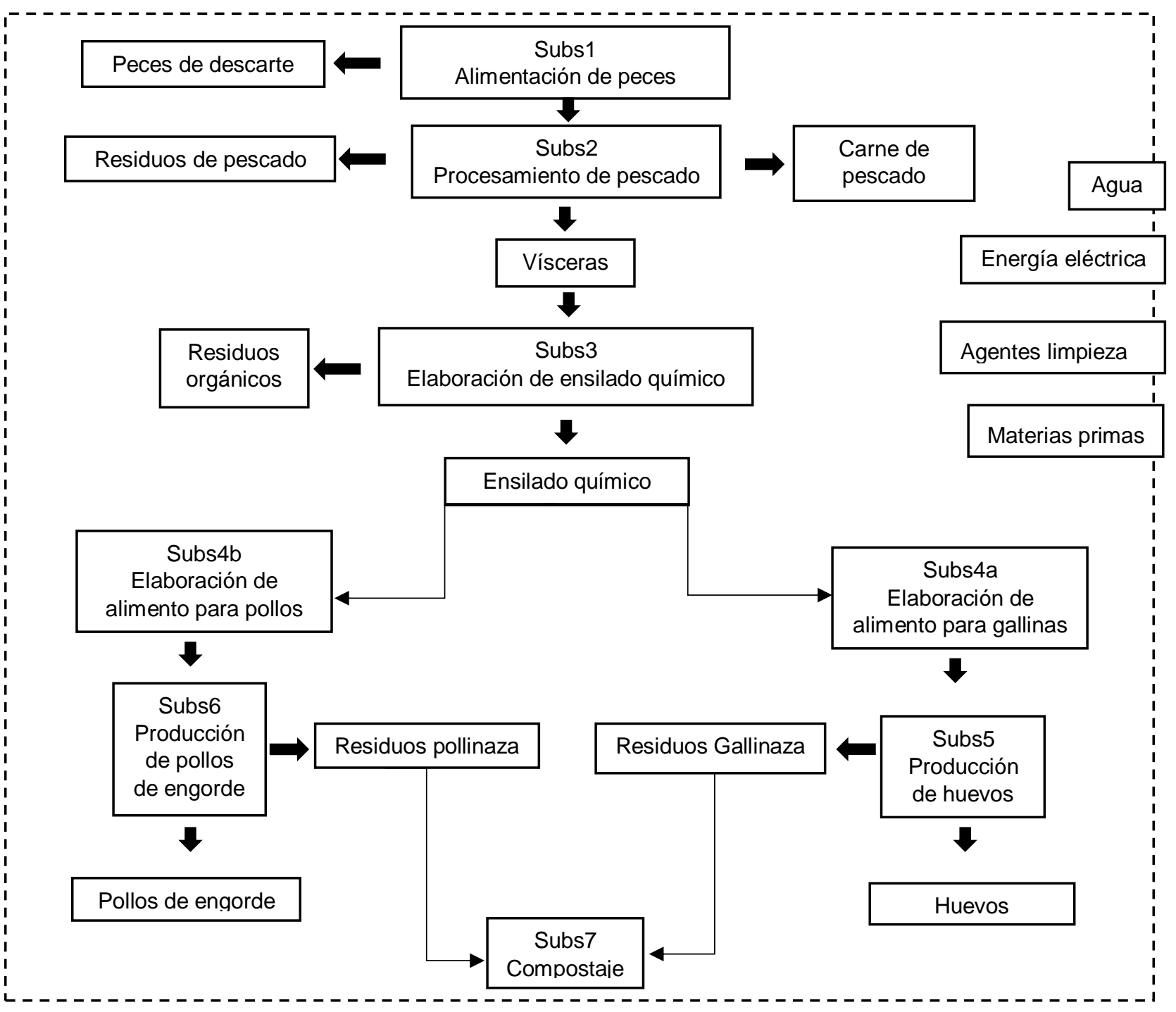

Fig. 1. Límite del sistema productivo con ensilado químico.

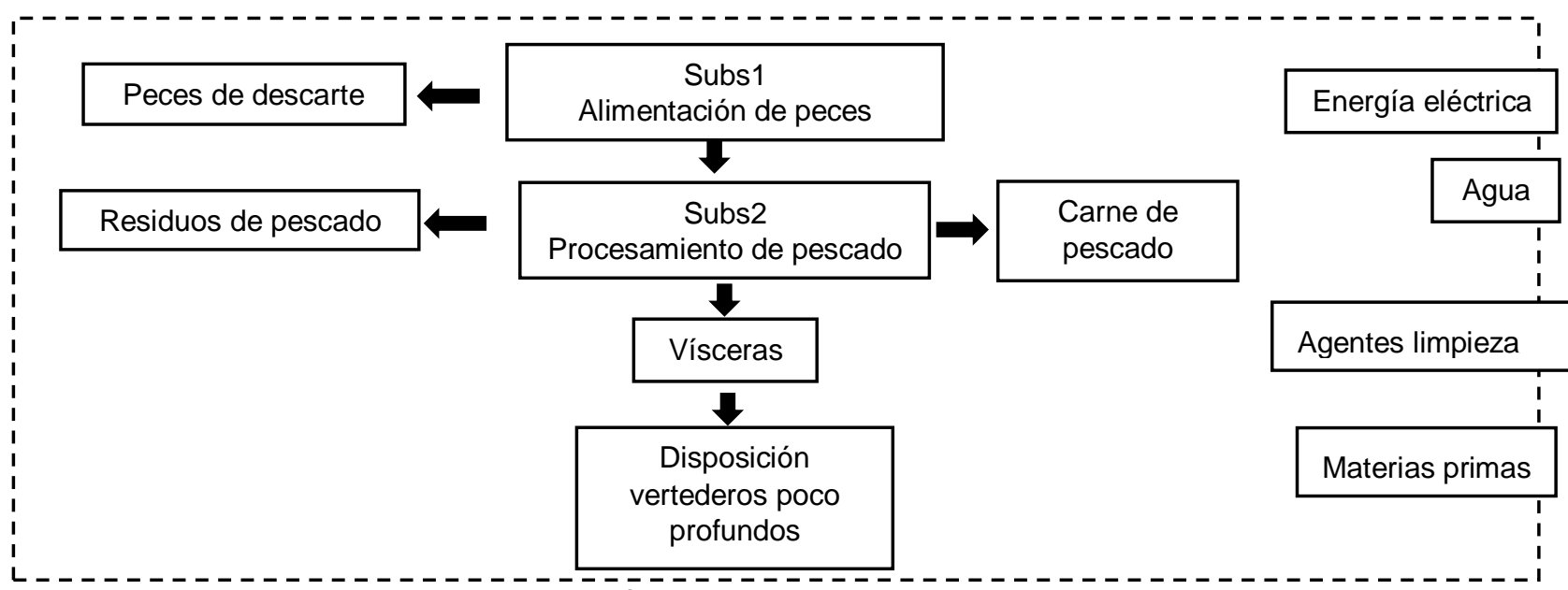

Fig. 2. Límite del proceso tradicional.

Subsistema 1 (Subs1): Hace referencia a la etapa de crianza y engorde de la tilapia roja (Oreochromis Ssp.), en el que se alimentaron 2044 alevinos durante un periodo de 7 meses hasta obtener un peso promedio de 400g. Los peces fueron alimentados con 3 dietas de concentrado comercial de acuerdo con etapa de crecimiento.

Subsistema 2 (Subs2): Consiste en el procesamiento de los peces. En esta etapa se procesaron 1778 peces correspondientes a una tasa de mortalidad del $13 \%$, donde los residuos de vísceras representaron el $16 \%$ del peso total obtenido. En el proceso tradicional estos residuos finalizan su ciclo siendo depositados en vertederos poco profundos. 
Subsistema 3 (Subs3): Corresponde al proceso de elaboración de ensilado químico (EQ) a partir de las vísceras, realizando etapas de desengrasado, triturado, ensilaje y almacenado según lo reportado por Suarez et al., (2018). El $69 \%$ del ensilado químico obtenido se destinó para la alimentación de gallinas ponedoras y $29 \%$ para alimentación de pollos de engorde.

Subsistema 4a y $4 b$ (Subs4a y Subs4b): Consiste en el proceso de elaboración de dietas tanto para gallinas ponedoras como para pollos de engorde. Este consta de tres etapas mezclado, pelletizado y secado. En la tabla 1 se observan las formulaciones definidas para la alimentación de ambas aves de acuerdo con los requerimientos nutricionales establecidos en las tablas brasileñas de nutrición. Adicionalmente se utilizó una premezcla de vitaminas y minerales la cual contenía la siguiente composición por $250 \mathrm{~g}$ de producto: vit. A $1,400,000$ IU; vit. B1 - 500 mg; vit. B12 - 300 mg; vit. B2 = 500 mg; vit. B6 - 1,6 g; vit. D3 - 2,500,000 IU; vit. $\mathrm{E}-6,000 \mathrm{IU}$; vit. $\mathrm{K} 3=1,000 \mathrm{mg}$; biotina - $30 \mathrm{mg}$; niacina $-12 \mathrm{~g}$; ácido fólico - $1 \mathrm{~g}$; cobalto - $50 \mathrm{mg}$; cobre 3,000 mg; hierro - 25 g; yodo - 500 mg; manganeso - 32.5 g; selenio - 100.50 mg; zinc - $22.49 \mathrm{~g}$.

Tabla 1. Formulación de las dietas para aves (\%)

\begin{tabular}{|l|c|c|}
\hline Materia prima & Gallinas ponedoras & Pollos de engorde \\
\hline Torta de soya & 8.10 & 9.35 \\
\hline Harina de pescado & 6.85 & 5.19 \\
\hline Ensilado & 21.91 & 21.76 \\
\hline Harina de maíz & 35.34 & 41.55 \\
\hline Harina de arroz & 13.06 & 17.66 \\
\hline Aceite de pescado & 1.00 & 0.85 \\
\hline Carbonato de calcio & 8.09 & 1.04 \\
\hline Fosfato de calcio & 4.04 & 1.04 \\
\hline Premezcla vitaminas & 0.40 & 0.52 \\
\hline Metionina & 0.30 & 0.26 \\
\hline Lisina & 0.30 & 0.26 \\
\hline Triptófano & 0.30 & 0.26 \\
\hline Treonina & 0.30 & 0.26 \\
\hline
\end{tabular}

ÇSubsistema 5 (Subs5): Hace referencia al proceso de obtención de huevos de gallina, para lo cual se alimentaron 36 gallinas ponedoras (Gallus gallus domesticus) de la raza Isa-Brown de 16 semanas de edad durante un periodo de 7 meses, sin embargo, para el análisis de resultados se tomó una base de cálculo en el tiempo, de un mes. Las gallinas fueron alimentadas 2 veces al día (mañana y tarde) con una ración de 107g por ave, teniendo un suministro constante de agua.

Subsistema 6 (Subs6): Se refiere al proceso de obtención de pollos de engorde, para lo cual se alimentaron 30 pollos (Gallus gallus domesticus) de la raza Ross 308 durante un periodo de 42 días hasta obtener un peso promedio de 1,684kg. Los pollos fueron alimentados 2 veces al día aumentando la ración diaria en función de la etapa de crecimiento, teniendo un suministro constante de agua.

Subsistema 7 (Subs7): Corresponde al proceso de compostaje de los residuos de excretas obtenidos en las etapas de alimentación de las aves.

\section{Suposiciones y limitaciones}

Para el presente estudio se tuvieron en cuenta algunos supuestos tales como: 1) El proceso de elaboración de EQ es llevado a cabo en la misma granja donde se crían los peces, para eliminar los efectos del transporte de las vísceras. 2) El impacto de las edificaciones no se tuvieron en cuenta en los cálculos debido a su que su tiempo de vida útil es extenso. 3) El impacto ambiental de los equipos industriales no se han incluido dentro del análisis ambiental, sin embargo, se ha demostrado que estos no afectan significativamente los resultados en la evaluación del impacto ambiental porque poseen altos periodos de vida útil (Perez-Martinez et al., 2018). 4) En el Subs4a y 4b se considera que los únicos compuestos liberados al ambiente debido al proceso de secado son moléculas de vapor de agua, ya que en esta etapa el sistema no alcanza temperaturas para la evaporación de otros compuestos.

\section{Cálculo del impacto ambiental}

Para el análisis del impacto ambiental del sistema productivo observado en la figura 1 se empleó el estudio de la huella ecológica. Esta metodología se llevó a cabo según lo establecido por Mathis Wackernagel y William Rees, (1996); Gwehenberger y Narodoslawsky, (2007); Krotscheck y Narodoslawsky, (1996), en los que se hace uso de modelos de cálculo que permiten estimar el impacto ecológico de cada etapa del sistema. Esto genera una mayor precisión en los resultados debido a que se requiere una alta recopilación de 
información y a su vez reduce las limitaciones que existen por el uso de bases de datos internacionales (Mamouni Limnios et al., 2009). Para el cálculo de huella ecológica del sistema se consideró como unidad fundamental la crianza de 2044 peces de la especie tilapia roja (Oreochromis Spp.). Los valores de los impactos ambientales de las entradas y salidas de cada etapa del sistema productivo fueron normalizados a una misma unidad ( $\mathrm{Ha} /$ ton) la cual indica el número de hectáreas de bosque necesarias en una región específica para fijar el $\mathrm{CO}_{2}$ producido durante cada etapa del sistema.

La ecuación 1 fue empleada para el cálculo del impacto ambiental (IA) ocasionado por los compuestos orgánicos (co) usados o generados dentro de cada etapa del sistema productivo. Este se realizó de acuerdo con lo establecido en las directrices del Intergovernmental Panel on Climate Change (IPCC), donde CRO corresponde a la cantidad del residuo orgánico, FCM corresponde al factor de corrección para el gas metano $\left(\mathrm{CH}_{4}\right)$, el cual depende del proceso asociado a la gestión de residuos sólidos en un sector en particular (IPCC, 2006), COD representa la fracción de carbono orgánico degradable del residuo que puede ser objeto de descomposición bioquímica (IPCC, 2006), CODF es la fracción de COD no asimilada o que se degrada muy lentamente (IPCC, 2006), $\mathrm{F}$ y R corresponden a la fracción de $\mathrm{CH}_{4}$ en el gas de vertedero y la fracción de $\mathrm{CH}_{4}$ recuperada respectivamente. El factor de oxidación del $\mathrm{CH}_{4}$ se representa como OX, el potencial de calentamiento global del metano PCG se usó para un periodo de 100 años (IPCC, 2006) y el FF corresponde al factor de fijación de $\mathrm{CO}_{2}$ para la región establecida dentro del análisis ambiental (IDEAM, 2016).

$$
\mathrm{IA}=\sum_{\mathrm{co}=1}^{\mathrm{n}} \mathrm{CRO}\left(\frac{16^{*}\left(\mathrm{FCM}^{*} \mathrm{COD}^{*} \mathrm{COD}_{\mathrm{F}}{ }^{*}(\mathrm{~F}-\mathrm{R})^{*}(1-\mathrm{OX})^{*} \mathrm{PCG}\right)}{12^{*} \mathrm{FF}}\right)
$$

El impacto ambiental proveniente de la entrada de energía eléctrica en las diferentes etapas se calculó mediante la ecuación 2, donde $\mathrm{EE}_{\mathrm{G}}$ corresponde al gasto de energía eléctrica "e" en cada etapa del proceso y EEE es el factor de emisiones de $\mathrm{CO}_{2}$ causadas por un kw-h de energía (IDEAM, 2016).

$$
\mathrm{IA}=\sum_{\mathrm{e}=1}^{\mathrm{n}} \mathrm{EE}_{\mathrm{G}} * \frac{\mathrm{EEE}}{\mathrm{FF}}
$$

En el subsistema 3 en la etapa de elaboración de ensilado químico se realiza un proceso de desengrasado mediante calentamiento con gas propano. Para determinar su impacto se realiza el cálculo usando la ecuación 3 , en donde $\mathrm{VR}_{\mathrm{C} 3 \mathrm{H} 8}$ es el volumen requerido de gas para el proceso, $\mathrm{EB}_{\mathrm{p}}$ es la energía embebida en el proceso de obtención de gas propano, la distancia recorrida en el transporte, el rendimiento de combustible y la carga del gas propano se denota $D_{R}, R_{c}$ y $C$ respectivamente, por ultimo las emisiones de $\mathrm{CO}_{2}$ provenientes del combustible se denominaron EC. Sumado a esto en dicho subsistema se tiene generación de $\mathrm{CO}_{2}$ a causa del proceso de combustión del gas propano para lo cual se calculó su impacto mediante la ecuación 4, en donde $\mathrm{FC}_{\mathrm{C} з \mathrm{H}}$ corresponde al factor de conversión para el gas propano y $\mathrm{FE}_{\mathrm{Сз}}$ es el factor de emisión de $\mathrm{CO}_{2}$ (IPCC, 2006).

$$
\begin{aligned}
& \mathrm{IA}=\mathrm{VR}_{\mathrm{C}_{3} \mathrm{H}_{8}}\left(\frac{\mathrm{EB}_{\mathrm{P}}{ }^{*} \mathrm{EEE}+\left(\frac{\mathrm{D}_{\mathrm{R}}}{\mathrm{R}_{\mathrm{C}}{ }^{*} \mathrm{C}}\right)^{*} \mathrm{EC}}{\mathrm{FF}}\right)
\end{aligned}
$$

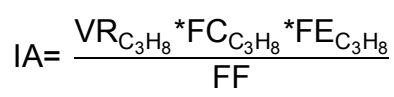

En los diferentes subsistemas es necesaria la inclusión de un flujo de entrada correspondiente al agua de limpieza tanto de equipos como de espacios destinados para los procesos. Para esto se calculó el impacto ocasionado por el suministro de agua mediante la ecuación 5 , en la cual $A_{L}$ corresponde a los litros de agua requerida en el proceso (a) y EB $\mathrm{B}_{\mathrm{A}}$ es la energía embebida para el suministro de agua. Sin embargo, el uso de esta trae consigo un impacto ambiental asociado a su disposición final, el cual es calculado mediante la ecuación 6 , donde $A R L$ corresponde a volumen de agua residual obtenida en el proceso de limpieza, COдBO5 es la demanda bioquímica de oxígeno de la fracción degradable del agua residual, y $\mathrm{CMP}_{\mathrm{CH} 4}$ indica la capacidad máxima de producción de metano que posee dicha fracción (IPCC, 2006). 


$$
\begin{aligned}
& \mathrm{IA}=\sum_{\mathrm{a}=1}^{\mathrm{n}} \mathrm{A}_{\mathrm{L}}\left(\frac{\mathrm{EB}_{\mathrm{A}}{ }^{*} \mathrm{EEE}}{\mathrm{FF}}\right) \\
& \mathrm{IA}=\sum_{\mathrm{a}=1}^{\mathrm{n}} \mathrm{AR}_{\mathrm{L}}{ }^{*}\left(\frac{\mathrm{CO}_{\mathrm{DBO} 5}{ }^{*} \mathrm{CMP}_{\mathrm{CH} 4}{ }^{*} \mathrm{FCM}_{\mathrm{CH} 4}{ }^{*} \mathrm{PCG}}{\mathrm{FF}}\right)
\end{aligned}
$$

Para los impactos ambientales asociados a los ingredientes de producción y demás flujos de entradas en los diferentes subsistemas se utiliza la ecuación 7, en donde $\mathrm{CR}_{1}$ es la cantidad requerida del producto $i$, EB $B_{\mathrm{PI}}$ corresponde a la energía embebida para la fabricación de este y $C_{1}$ la carga máxima en el transporte del producto (IPCC, 2006).

$$
I A=C R_{1}\left(\frac{E B_{P \mid}{ }^{*} E E E+\left(\frac{D_{R}}{R_{C}{ }^{*} C_{l}}\right){ }^{*} E C}{F F}\right)
$$

\section{RESULTADOS Y DISCUSIÓN}

En la tabla 2 se presentan la ganancia de peso de los pollos y el porcentaje de postura de las gallinas. Se observa que el peso de los pollos mostró una tendencia creciente durante todo el tiempo del estudio, mientras que sus valores están en el rango típico para esta línea de aves (Ross 308) (Rosero et al., 2012). Es así como Boitai et al., (2018), obtuvieron pesos de 1701 gr y 1660 gr respectivamente, en pollos de engorde alimentados a partir de dietas con sustitución parcial (5\% y 10\%) de la fuente de proteína por ensilado químico de vísceras y branquias de peces de agua dulce, concluyendo que la sustitución no presento efectos adversos sobre esta variable productiva. Del mismo modo, Al-Marzooqi et al., (2010), demostraron que reemplazar harina de soya con proteína de ensilado de pescado, mejoró el rendimiento de crecimiento de los pollos de engorde. Sin embargo, se debe destacar que en el presente trabajo la sustitución de la fuente de proteína utilizada fue del $21,76 \%$ (tabla 1), con lo que se incrementa la recuperación del residuo, sin afectar el desempeño productivo de las aves.

En cuanto al porcentaje de postura (tabla 2), se puede observar que mantiene valores estables en torno al $85 \%$, lo que indica que el uso de ensilado desengrasado en la alimentación de las gallinas ponedoras en el presente estudio no tuvo efectos adversos sobre el porcentaje de postura, señalando que las características nutricionales de las dietas estuvieron ajustados a los requerimientos de las aves. Resultados similares fueron encontrados por Kjos et al., (2001), sin embargo, en dicho estudio se usó 5 \% de ensilado.

Tabla 2. Parámetros productivos de las aves

\begin{tabular}{|l|c|c|}
\hline Semanas & $\begin{array}{c}\text { Ganancia de Peso } \\
\text { pollos de engorde }(\mathrm{g})\end{array}$ & $\begin{array}{c}\text { Porcentaje de } \\
\text { Postura gallinas }\end{array}$ \\
\hline $0-1$ & $42.10 \pm 3.42$ & $82.14 \pm 4.30$ \\
\hline $1-2$ & $114.14 \pm 10.94$ & $85.71 \pm 5.81$ \\
\hline $2-3$ & $262.96 \pm 27.68$ & $82.14 \pm 4.53$ \\
\hline $3-4$ & $500.43 \pm 49.63$ & $85.71 \pm 2.76$ \\
\hline $4-5$ & $780.52 \pm 69.85$ & $85.71 \pm 4.69$ \\
\hline $5-6$ & $1278.56 \pm 91.27$ & $100.00 \pm 3.26$ \\
\hline $6-7$ & $1684.10 \pm 58.72$ & $89.29 \pm 3.18$ \\
\hline
\end{tabular}

\section{Impacto ambiental del uso de ensilado}

Los buenos resultados obtenidos con el uso de ensilado, justifica que se haga el análisis del impacto ambiental de su utilización. Para tal efecto, la cuantificación del impacto ambiental y la cantidad del producto o residuo obtenido en cada uno de los subsistemas del proceso (Fig. 1), se presenta en la tabla 3. Con respecto al subsistema 1 (Crianza de peces), la mortalidad de los peces generó una reducción del 13\% de la población inicial (2044 peces), lo cual está en el rango típico de un ciclo productivo de esta naturaleza (10-20\%). Dicho subsistema presenta el mayor impacto ambiental de todo el sistema productivo $(0,574878$ ha/cycle), debido a la gran cantidad de alimento consumido por los peces en su crianza ( 7 meses), que en el caso de la tilapia roja tiene requerimientos de flotabilidad y el cultivo de algunas materias primas para cumplir con este requerimiento, están asociados con emisiones de gases de efecto invernadero (Henriksson et al., 2015). 
En la etapa del procesamiento del pescado (Subs 2), se retiran las escamas (5\%) y las vísceras (16\%), entregando un rendimiento promedio en peso del $79 \%$. Esta etapa presenta un impacto ambiental considerablemente mayor, respecto a las etapas posteriores, debido a los altos volúmenes de agua requeridos durante el eviscerado, además de la elevada carga orgánica presente en las vísceras, las cuales en la mayoría de los casos se vierten al ambiente (Quiroz Fernández et al., 2018).

En el proceso de obtención de ensilado químico correspondiente al subs 3, se obtiene un rendimiento de $71 \%$ luego del desengrasado, equivalente a $80,73 \mathrm{~kg}$ de EQ, el cual es usado posteriormente en la elaboración de las dietas para pollos de engorde y gallinas ponedoras. Con respecto al impacto ambiental en esta etapa se observa un valor de -0,1440 ha/ciclo, el cual presenta signo negativo debido a que este corresponde al aprovechamiento de un subproducto (vísceras) que de no ser usado generaría un impacto ambiental equivalente a esta magnitud. Se debe tener en cuenta que en el presente estudio se tomó como base de cálculo una granja de tamaño moderado, por lo que se debe considerar el potencial que tiene este modelo si se lleva a granjas de mayor tamaño. Comportamientos similares fueron reportados por Malakahmad et al., (2017), quienes determinaron el impacto ambiental de diferentes procesos de disposición de residuos orgánicos, encontrando que las emisiones de $\mathrm{CO}_{2}$ que fueron evitadas para el proceso de digestión anaerobia son considerablemente mayores a las emisiones de $\mathrm{CO}_{2}$ totales del proceso por lo tanto esto da como resultado un valor negativo, lo que significa que se está favoreciendo el medio ambiente con dicho proceso. Es así como la revaloración de los residuos de la industria piscícola comparando con el proceso tradicional de disposición de residuos indica que el proceso de obtención de ensilado químico permite mitigar aproximadamente el $30 \%$ de las emisiones de $\mathrm{CO} 2$, con respecto a las generadas en el ambiente cuando se descargan las vísceras frescas en vertederos poco profundos, generando un crédito de huella ecológica por revaloración de residuos agroindustriales.

Los subsistemas $4 \mathrm{a}$ y $4 \mathrm{~b}$ correspondientes al proceso de elaboración del concentrado para alimentación de las gallinas y los pollos respectivamente, presentan impactos ambientales relativamente bajos en comparación con las demás etapas del proceso y sus principales categorías de impacto se centran en la obtención de las materias primas y la energía eléctrica usada en el proceso de pelletización. El proceso de secado del producto se realizó mediante el uso de colectores solares, como fue reportado por Camaño et al., (2020), para disminuir la carga ambiental proveniente del alimento, con lo cual este trabajo se enmarca en la tendencia del uso de energías renovables en los procesos de secado de alimentos balanceados, ya que la evaluación del desempeño ambiental de diferentes granjas y plantas de procesamiento de carne de pollo, han mostrado que uno de los principales perfiles ambientales de las granjas avícolas es la producción de piensos, por su alto consumo energético y el uso de granos como la semilla de soya, que requieren la aplicación de fertilizantes minerales (Skunca et al., 2018). El alto impacto de la torta de soja en los piensos se debe en gran parte a los gases de efecto invernadero liberados como resultado de los cambios en el uso de la tierra (Leinonen et al., 2012a). Adicionalmente, el aprovechamiento de los residuos de la industria piscícola como sustituto parcial de materias primas proteicas, genera una reducción de la demanda de este tipo de materias primas, las cuales a su vez poseen impactos ambientales de importancia en su proceso de producción, como es el caso de la soya (Leinonen et al., 2012b).

Tabla 3. Impacto ambiental de los subsistemas

\begin{tabular}{|l|c|c|}
\hline \multicolumn{1}{|c|}{ Subsistema } & Cantidad & $\mathrm{IA}(\mathrm{Ha} /$ ciclo) \\
\hline Subs 1 $(\mathrm{U})$ & 1778,28 & 0,5748 \\
\hline Subs 2 $(\mathrm{Kg})$ & 561,94 & 0,4684 \\
\hline Subs 3 $(\mathrm{Kg})$ & 80,73 & $-0,1440$ \\
\hline Subs 4a $(\mathrm{Kg})$ & 115,05 & 0,0755 \\
\hline Subs 4b $(\mathrm{Kg})$ & 105,47 & 0,0543 \\
\hline Subs 5 $(\mathrm{U})$ & 972,00 & 0,1633 \\
\hline Subs 6 $(\mathrm{Kg})$ & 50,52 & 0,1465 \\
\hline Subs 7 $(\mathrm{Kg})$ & 211,20 & $-0,1138$ \\
\hline \multicolumn{2}{|r|}{ Total } & 1,2251 \\
\hline
\end{tabular}

El factor de conversión alimenticio ( $\mathrm{FCl}$ ), es una variable de importancia ambiental en los procesos de producción de huevo o carne, puesto que las especies avícolas emiten gases de efecto invernadero como el metano, durante la digestión y esta variable relaciona la cantidad de gases producidos por unidad de alimento consumido, para una unidad carne o huevo producida (Ibidhi et al., 2017). Para los subsistemas 5 y 6 los valores del impacto ambiental en la alimentación de las aves se ven influenciados fundamentalmente por el consumo del alimento, pues este afecta la cantidad de estiércol generado y la composición de este, influyendo en las emisiones del sistema productivo avícola (crecimiento y obtención de productos). Comportamientos similares fueron reportadas por Leinonen et al., (2012), quienes concluyeron que los cambios en el consumo y la composición del alimento tienen efectos tanto en los impactos ambientales que ocurren durante la 
producción del cultivo y el procesamiento del alimento, como en las emisiones subsecuentes del estiércol de aves de corral durante el alojamiento, almacenamiento y aplicación en el campo.

Las excretas de aves de corral son la fuente de emisiones gaseosas directas de amoníaco (NH3), óxido nitroso (N2O) y en menor medida el metano $(\mathrm{CH} 4)$, que se produce durante el periodo productivo, el almacenamiento y esparcimiento en la tierra (Leinonen et al., 2012b). En algunas de las etapas, como en el subsistema 7, el estiércol tuvo un valor negativo; es decir, fue un crédito en lugar de una carga ambiental. Esto se debió al uso de las excretas como fertilizante, que compensó la producción de fertilizantes sintéticos o cultivos rotativos fijadores de nitrógeno.

Las principales categorías de impactos a la entrada y la salida de los subsistemas del sistema productivo se pueden observar en la figura 3 , donde se destacan las principales fuentes de emisiones de gases de efecto invernado. El uso de energía eléctrica se caracteriza por ser uno de los indicares de mayor impacto en cuanto a la cuantificación ambiental (Tian et al., 2012).

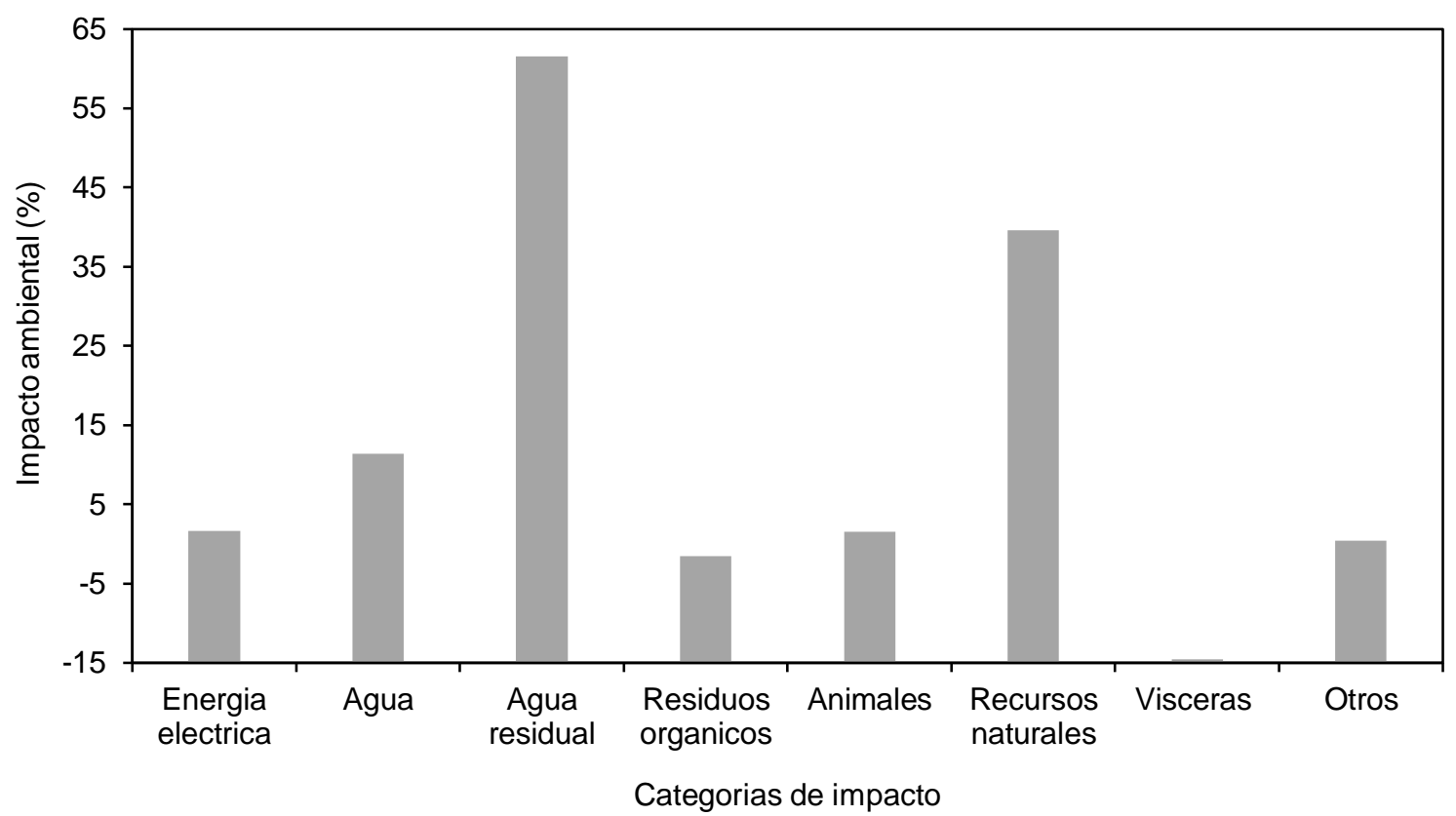

Fig. 3 Porcentaje representación por categorías para el sistema productivo completo

En este estudio el uso de energías alternativas como secado solar en el proceso de elaboración del alimento para las especies avícolas, genero una disminución significativa de las emisiones de $\mathrm{CO}_{2}$ en dicha categoría, esto se debe a que la energía solar usada es una fuente abundante, renovable y gratuita, convirtiéndola en una de las alternativas más prometedoras para disminuir el impacto ambiental de los procesos de secado (Tiwari, 2016). Con respecto al proceso de disposición del agua residual, se observa que es la categoría con mayor impacto ambiental del sistema productivo $(61 \%)$, fundamentalmente por la carga orgánica presente la cual genera una dificultad en el proceso de su disposición puesto que requiere mayor cantidad de oxígeno para su degradación, reduciendo la capacidad de esta para asimilar carga contaminante y restituir su calidad de forma natural (Quiroz et al., 2018). El aprovechamiento de residuos orgánicos tanto de la industria piscícola (vísceras) como avícola (excretas), favorecen la reducción del impacto ambiental de forma directa, además de que se genera un valor agregado a los subproductos que repercute en la economía del proceso. El uso de recursos naturales para el cultivo de las materias primas e insumos necesarios para la elaboración de los alimentos tanto acuícolas como avícolas, representa la segunda fuente del impacto ambiental del sistema productivo con un $39,59 \%$, principalmente por el hecho de que la soya es uno de los principales potenciadores de calentamiento global (GWP100) (Leinonen et al., 2012b).

\section{CONCLUSIONES}

El desarrollo productivo de las especies avícolas usadas en el presente estudio, no se ve afectado negativamente por el uso de ensilado químico de vísceras de tilapia roja (Oreochromis Spp.), como sustituto de fuentes proteicas convencionales. El impacto ambiental del sistema productivo el uso de recursos naturales para la elaboración de concentrados para las industrias acuícolas y avícolas y el tratamiento de aguas residuales son las principales fuentes de efectos adversos al ambiente, sin embargo, el aprovechamiento de subproductos de ambas industrias y la implementación de energías alternativas renovables y eficientes reducen significativamente la carga ambiental del proceso, además de generar productos con valor comercial. 


\section{AGRADECIMIENTOS}

Los autores del presente trabajo agradecen a la Universidad de Antioquia y a COLCIENCIAS (proyecto 1115745-58746) por el apoyo financiero brindado para su desarrollo.

\section{NOTACION}

Símbolos

$\mathrm{FCM}=$ Factor de corrección del metano

$\mathrm{COD}=$ Carbono orgánico degradable

$\mathrm{COD}_{\mathrm{F}}=$ Carbono orgánico degradable no asimilada

$\mathrm{F}=$ Fracción de metano en el gas de vertedero

$\mathrm{R}=$ Fracción de metano recuperada

$\mathrm{OX}=$ Factor de oxidación del metano

$\mathrm{PCG}=$ Potencial de calentamiento global

$F F=$ Factor de fijación

$E_{\mathrm{G}}=$ Gasto de energía eléctrica

$\mathrm{EEE}=\mathrm{Factor}$ de emisión de $\mathrm{CO}_{2}$ por $\mathrm{Kw}$ de energía

$\mathrm{VR}_{\mathrm{C} 3 \mathrm{H} 8}=$ Volumen de gas propano

$\mathrm{EB}_{\mathrm{P}}=$ Energía embebida producción de propano

$D_{R}=$ Distancia recorrida

$\mathrm{R}_{\mathrm{C}}=$ Rendimiento de combustible

$\mathrm{C}=$ Carga de combustible

$\mathrm{EC}=$ Emisiones de combustible

$\mathrm{FC}_{\mathrm{C} 3 \mathrm{H} 8}=$ Factor de conversión de gas propano

$\mathrm{FE}_{\mathrm{C} 3 \mathrm{H} 8}=$ Factor de emisión

$\mathrm{AL}=$ Volumen de agua

$\mathrm{EB}_{\mathrm{a}}=$ Energía embebida del agua

$\mathrm{AR}_{\mathrm{L}}=$ Volumen de agua residual

$\mathrm{CO}_{\mathrm{DBO} 5}=$ Demanda bioquímica de oxígeno del agua residual

$\mathrm{CMP}_{\mathrm{CH} 4}=$ Capacidad máxima de producción de metano

$\mathrm{CR}=$ Cantidad requerida de producto

$\mathrm{EB}_{\mathrm{Pi}}=$ Energía embebida en la fabricación

$\mathrm{C}_{\mathrm{i}}=$ Carga máxima en el transporte del producto

\section{Abreviaciones}

Subs= Subsistema

$\mathrm{EQ}=$ Ensilado químico

IA = Impacto ambiental

IPCC= Intergovernmental Panel on Climate Change

$\mathrm{CO}=$ Compuesto organico

$\mathrm{CRO}=$ Cantidad de residuos organico

\section{REFERENCIAS}

Ahmed, Z., y Wang, Z., Investigating the impact of human capital on the ecological footprint in India: An empirical analysis. https://doi.org/10.1007/s11356-019-05911-7, Environ. Sci. Pollut. Res., 26(26), 26782-26796 (2019)

Al-Marzooqi, W., Al-Farsi, M. A., y otros tres autores., The effect of feeding different levels of sardine fish silage on broiler performance, meat quality and sensory characteristics under closed and open-sided housing systems. https://do.org/10.5713/ajas.2010.10119, Asian-Aust. J Anim Sci., 23(12), 1614-25 (2010)

Boitai, S. S., Lakshman, K. B., y otros cinco autores., Effect of dietary incorporation of fish silage on growth performance, serum biochemical parameters and carcass characteristics of broiler chicken. https://do.org/10.18805/ijar.B-3315, Indian J. Anim. Res., 52(7),1005-9 (2018)

Camaño, J. A., Rivera, A. M., y Zapata, J. E. Efecto del espesor de película y de la ubicación de la muestra en un secador solar directo, sobre la cinética de secado de ensilado de vísceras de tilapia roja (Oreochromis sp). https://doi.org/10.4067/s0718-07642020000100053, Inf. Tecnol., 31(1), 53-66 (2020)

Gomez, G., Ortiz, M., y otros dos autores., Evaluación del ensilaje de vísceras de tilapia roja (Oreochromis Spp) en alimentación de pollos de engorde. biotecnología en el sector agropecuario y agroindustrial, 12(1), 106-14 (2014)

Gwehenberger, G., y Narodoslawsky, M., The sustainable process index SPI as an engineer's tool for environmental impact assessment of processes: The sugar industry as a case study. https://doi.org/10.2495/SDP070321, WIT Trans. Ecol. Environ., 102, 333-340 (2007)

Gwehenberger, G., y Narodoslawsky, M., The ecological impact of the sugar sector- Aspects of the change of a key industrial sector in Europe. https://doi.org/10.1016/S1570-7946(07)80196-9, Comput. Aided Chem. Eng., 24, 1029-1034 (2007) 
Henriksson, P., Rico, A., y otros dieciocho autores., Comparison of Asian aquaculture products by use of statistically supported life cycle assessment. https://doi.org/10.1021/acs.est.5b04634, Environ. Sci. Technol., 49(24), 14176-14183 (2015)

Ibidhi, R., Hoekstra, A., y otros dos autores., Water, land and carbon footprints of sheep and chicken meat produced in Tunisia under different farming systems. https://doi.org/10.1016/j.ecolind.2017.02.022, Ecol. Indic., 77, 304-313 (2017)

IDEAM. Inventario nacional y departamental de gases de efecto invernadero - Colombia. (http://documentacion.ideam.gov.co/openbiblio/bvirtual/023634/INGEl.pdf) (2016)

IPCC. Directrices para los inventarios nacionales de gases de efecto invernadero - Elimimación de desechos sólidos Capitulo 3. (2006)

Jóhannesson, S., Davíðsdóttir, B., y Heinonen, J., Standard ecological footprint method for small, highly specialized economies. https://doi.org/10.1016/j.ecolecon.2017.11.034, Ecol. Econ., 146(December 2016), 370-380 (2018)

Kjos, N., Herstad, O., y otros dos autores., Effects of dietary fish silage and fish fat on performance and egg quality of laying hens. https://doi.org/10.4141/A00-086, Can. J. Anim. Sci., 81(2), 245-251 (2001)

Krotscheck, C., y Narodoslawsky, M., The sustainable process index. A new dimension in ecological evaluation. https://doi.org/10.1016/0925-8574(95)00060-7, Ecol. Eng., 6(4), 241-258 (1996)

Leinonen, I., Williams, A. G., y otros tres autores., Predicting the environmental impacts of chicken systems in the United Kingdom through a life cycle assessment: Broiler production systems. http://dx.doi.org/10.3382/ps.2011-01634, Poult. Sci. J. 91 (8-25) (2012)

Leinonen, I., Williams, A. G., y otros tres autores., Predicting the environmental impacts of chicken systems in the united kingdom through a life cycle assessment: Egg production systems. https://doi.org/10.3382/ps.2011-01635 Poult. Sci. J. $9126-40(2012 b)$

Malakahmad, A., Abualqumboz, M., y otros dos autores., Assessment of carbon footprint emissions and environmental concerns of solid waste treatment and disposal techniques; case study of Malaysia. https://doi.org/10.1016/j.wasman.2017.08.044, Waste Manage., 70, 282-292 (2017)

Mamouni, E., Ghadouani, A., y otros dos autores., Giving the consumer the choice: A methodology for Product Ecological Footprint calculation. https://doi.org/10.1016/j.ecolecon.2009.04.020, Ecol. Econ., 68(10), 2525-2534 (2009)

Martínez-Alvarez, O., Chamorro, S., y Brenes, A., Protein hydrolysates from animal processing by-products as a Source of bioactive molecules with interest in Animal feeding: A review, https://do.org/10.1016/j.foodres.2015.04.005, Food Res. Int., 73 (1069) 204-212 (2015)

Perez, M., Noguerol, R., y otros tres autores., Evaluation of environmental impact of two ready-to-eat canned meat products using Life Cycle Assessment. https://doi.org/10.1016/j.jfoodeng.2018.05.031, J. Food Eng., 237(May), 118-127 (2018)

Quiroz, L., Izaquierod, E., y Menéndez, C., Estudio del impacto ambiental del vertimiento de aguas residuales sobre la capacidad de autodepuración del río Portoviejo, Ecuador. Centro Azúcar, 45(01), 73-83 (2018).

Rosero, J. Ferney, E., y Lopez, F,. Evaluación del comportamiento productivo de las lineas de pollos de engorde Cobb 500 Y Ross 308. Biotecnología En El Sector Agropecuario y Agroindustrial, 10(1):8-15. (2012).

Sharif, A., Baris-Tuzemen, O., y otros tres autores., Revisiting the role of renewable and non-renewable energy consumption on Turkey's ecological footprint: Evidence from Quantile ARDL approach.

https://doi.org/10.1016/j.scs.2020.102138, Sustain. Cities Soc., 57(August 2019), 102138 (2020)

Skunca, D., Tomasevic, I., y otros tres autores., Life cycle assessment of the chicken meat chain. https://doi.org/10.1016/j.jclepro.2018.02.274, J. Clean. Prod., 184, 440-450 (2018)

Suarez, L. M., Montes, J. R., y Zapata, J. E. Optimización del contenido de acidos en ensilados de vísceras de tilapia Roja (Oreochromis spp.) con análisis del ciclo de vida de los alimentos derivados, https://do.org/10.4067/s071807642018000600083, Inf. Tecnol., 29(6), 83-94 (2018)

Syrovátka, M., On sustainability interpretations of the Ecological Footprint. https://doi.org/10.1016/j.ecolecon.2019.106543, Ecol. Econ., 169(December 2019), 106543 (2020)

Tian, M., Gao, J., y otros dos autores., The study on the ecological footprint of rural solid waste disposal-example in Yuhong district of shenyang. https://doi.org/10.1016/j.proenv.2012.10.013, Procedia Environ. Sci. Eng. Manag., 16(0), 95-101 (2012)

Tiwari, A., A review on solar drying of agricultural produce. https://doi.org/10.4172/2157-7110.1000623, J. Food Process. Preserv., 7(9) (2016)

Wackernagel, M., y Rees, W., Review: Our ecological footprint: reducing human impact on the Earth., New Society Publishers., 160 (1996) 